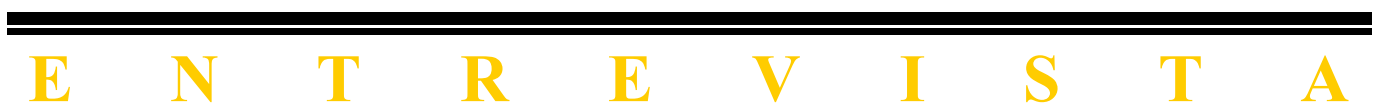




\section{ENTREVISTA A AULIS AARNIO}

Manuel Atienza: En el número 1 de Doxa, en 1984, distinguías tres etapas en tu trabajo como filósofo del Derecho: la primera, durante la década de los 60, habría estado dedicada a la elucidación de algunos conceptos de la dogmática jurídica; la segunda, que comprendería la primera mitad de los 70, a la explicación intencional de la conducta jurídica; y la tercera, a la justificación de las decisiones jurídicas. ¿Cabría hablar de una cuarta, o quinta, etapa en tu obra teórica?

Aulis Aarnio: En los años 90, la teoría de la argumentación jurídica ha estado, de alguna forma, en el trasfondo de mi obra. Este cambio quizás pudiera caracterizarse diciendo que, como filósofo del Derecho, estoy más interesado en las precondiciones de la argumentación (de la justificación) que en la teoría de la argumentación en sí misma. Con lo de «precondiciones» me refiero, por ejemplo, a las bases ontológicas del pensamiento jurídico. ¿En qué sentido específico existen las instituciones jurídicas? Esta cuestión no está contestada por la teoría jurídica institucional de Neil MacCormick y Ota Weinberger. Por el contrario, el convencionalismo que, entre otros, representa Eeerik Lagerspetz parece ofrecer posibilidades atractivas.

Relacionado en parte con lo antes mencionado, he empezado a reflexionar sobre la cuestión de la función del Derecho en la constitución de las relaciones sociales o, en último término, en la constitución de la sociedad. La tarea del Derecho consiste en fortalecer la integración social (y la socialización). Es ésta la razón de que para mí sea más importante investigar sobre cómo es que las normas jurídicas forman la base de la sociedad, más bien que estudiar cuál es su estructura o cómo pueden ser representadas lógicamente.

Ésta es la razón por la que he tratado, más que anteriormente, de tomar en consideración la naturaleza societaria del Derecho. Sin embargo, al reflexionar sobre ello, no he utilizado los conceptos que se usaron hasta el final de los 80. En mi opinión, las teorías colectivistas, tal como los diversos 
modelos marxistas, por ejemplo, no ofrecen un fundamento aceptable para comprender la sociedad de comienzos del siglo XXI. Por un lado, ha aumentado el dominio de las fuerzas del mercado y, como resultado de ello, se ha remarcado el individualismo extremo, como dicen los post-modernistas. Muchos pensadores neoliberales y sus seguidores no teóricos sostienen que es imposible dirigir la sociedad -que se desarrolla de acuerdo con las fuerzas del mercado.

No me gustaría ceder a este tipo de concepciones deterministas, con independencia de si las ideas vienen de concepciones colectivistas o de la creencia en las fuerzas del mercado. Baso mi concepción en la idea de que existe una «tercera vía», mediante la cual pueden evitarse ambos extremos. El Derecho tiene una importante tarea en el diseño teórico de la tercera vía. En cualquier caso, es un desafío al Derecho.

Ésta ha sido también una razón esencial por la que se fundó la revista Associations (Dunker \& Humblot, Berlín). Como editor en jefe, suscribo el objetivo básico señalado en la comunicación de la fundación de la revista, esto es, la búsqueda de alternativas a la situación socio-política prevalente.

La idea de la tercera vía ha tenido también otro tipo de impacto en mi pensamiento. Considero a la ciencia jurídica como una de las ciencias sociales esenciales, incluso más fundamental o al menos tan fundamental como la sociología teórica. Aquí es una cuestión del diseño teórico de la sociedad, porque la sociedad toma su forma siempre y en todos los lados a través del Derecho. En su parte fundamental, el Derecho conforma aquellas relaciones sociales cuya red teórica es la misma que la de la sociedad.

En años recientes, en mis escritos he remarcado especialmente este rol de la ciencia jurídica. Y si el problema se contempla de esta forma, la teoría jurídica tiene mucho en común con la sociología teórica (no con la sociología empírica) y con la teoría política. Ambas analizan la construcción de la sociedad.

Quizás hayan sido estas ideas las que me han llevado a mostrar un renovado interés por la teoría de la acción de Georg Henrik von Wright, en la que estuve muy interesado en los años 70. En aquel momento, examinaba fundamentalmente la posibilidad de comprender la acción de un actor individual, por ejemplo, la conducta de un juez, sobre la base de su motivación. Hoy en día, me ocupo más, por así decirlo, de las acciones colectivas. ¿Qué es una acción jurídica, en cuanto acción colectiva? ¿Cómo funciona la teoría de las acciones colectivas en la interpretación de las instituciones sociales? He examinado estos problemas en dos de mis artículos recientemente publicados (un artículo en Ratio Juris, de 1998, y un trabajo en el Festschrift a Georg Henrik von Wright, editado por Georg Meggle en 1998). 
M. A.: ¿Cuáles consideras que han sido tus principales contribuciones a la teoría del Derecho desde 1984?

A.A.: Desde 1984, mis principales contribuciones han estado vinculadas a la teoría de la argumentación, y durante el último o los dos últimos años, a la filosofía social en el sentido antes mencionado.

Las obras principales referidas a la teoría de la argumentación son «The Rational as Reasonable» (Kluwer, 1987), que ha sido traducida al castellano por el Centro de Estudios Constitucionales; y «Reason and Authority» (Ashgate, 1997). Además he publicado docenas de artículos sobre el tema en varias lenguas.

En cuanto a los contenidos, lo que quizás valoro más -si se me permite hablar asíson algunas de mis observaciones sobre ontología del Derecho, como, por ejemplo, las publicadas en el Festschrift a Ernesto Garzón Valdés. También se relacionan con esto algunas de mis interpretaciones de Wittgenstein; especialmente, el concepto de «forma de vida» y otros conceptos relacionados con él. Quizás haya sido también capaz de decir algo importante en relación con el punto de vista procedimental en el pensamiento jurídico, sobre lo que también Robert Alexy ha escrito mucho. Y todo ello está vinculado con los análisis que conciernen a la legitimidad del Derecho y de la actividad jurídica.

M. A.: ¿Cuáles son tus intereses y tus proyectos iusfilosóficos en el momento presente?

A. A.: Sobre mis intereses en este momento en filosofía del Derecho, se desprenden claramente de mi contestación a la primera pregunta. Hablando en general -y repitiendo lo antes mencionado- el foco de interés de mi pensamiento se ha trasladado drásticamente hacia la filosofía social. En su sentido técnico, la teoría de la argumentación no es ya, en mi opinión, tan interesante como acostumbró a serlo; por ejemplo, la teoría de las normas difícilmente tiene algo nuevo que ofrecerme.

Si mis fuerzas me lo permiten, planeo escribir en los próximos anos algo así como una síntesis de mi pensamiento. Naturalmente, se basará en mis escritos previos, pero el énfasis en la filosofía social resultará sustancialmente más evidente que en cualquiera de los escritos que he publicado hasta ahora.

M. A.: Hemos hablado indistintamente de teoría del Derecho y de filosofía del Derecho. ¿Cómo ves la situación actual de nuestra disciplina, cualquiera que sea el nombre que se le quiera dar?

A. A.: La posición de la teoría jurídica, al menos en los países nórdicos, no ha llegado a ser tan sólida como en los años 80 pensábamos que llegaría a ser. Muchas disciplinas jurídicas no teoréticas han logrado una firme posición en las Facultades de Derecho. A pesar de ello, diría que la teoría jurídica 
deberá hacer frente, en el siglo XXI, a un importante desafío. El desafío lo planteará el desarrollo de Europa o, por qué no, también el desarrollo a nivel mundial.

Por un lado, se está produciendo una fuerte globalización y, por otro lado, una fuerte tendencia al localismo (en Europa, el regionalismo). También las fuerzas del mercado están incrementando su poder a costa de las democracias. Los políticos apelan cada vez más al hecho de que el espacio de la política se está empequeñeciendo. Como dice John Kenneth Galbraith, predomina «una tiranía de las circunstancias».

Una de las tareas esenciales de la filosofía social y jurídica consiste en examinar si esto es o no cierto. De manera específica, la filosofía jurídica, al ser un área clave de la filosofía social, tendrá que hacer frente a este desafío. Una vez más, hago referencia a lo que he dicho sobre la tercera vía. La filosofía jurídica debe ser capaz de asumir conceptualmente el papel del Derecho en una realidad cambiante. Anticipo que, en este proceso, los medios teóricos del siglo XX (lógica deóntica, teoría de las normas, teoría de la argumentación) no pueden ser utilizados en el siglo XXI, de la forma como fue todavía posible en los años 50 cuando empecé a investigar en filosofía jurídica.

Georg Henrik von Wright escribió en una ocasión, refiriéndose al desarrollo social antes mencionado, que necesitaríamos un nuevo Marx para analizar la situación social imperante. La teoría del nuevo Marx. no podría ser la misma que la de Karl Marx, pero tendría la misma función y tarea. La anti -socialización y la desintegración en las relaciones sociales, por ejemplo, no pueden continuar indefinidamente. En uno u otro estadio, comenzará un nuevo tipo de socialización, pero probablemente no estará basado en ninguna teoría que resulte hoy visible. Será alguna otra cosa, la tercera vía. ¿Pero qué puede ser? No soy capaz de imaginarlo. La sociedad es demasiado no-transparente para poder hacerlo.

Sin embargo, creo que en el futuro habrá una cuestión que resultará más visible de lo que hoy es: la dirección de la sociedad con medios democráticos. Y ello, por su lado, llevará a una situación en la que las cuestiones de valores (morales) importarán más que en el presente. Las sociedades y las instituciones tendrán que tomar postura acerca de qué es lo mejor del hombre en el mundo. Y una vez más, se trata de cuestiones fundamentales de la filosofía jurídica. Como todos sabemos, la filosofía jurídica ha tratado siempre con cuestiones de valores. A veces, los valores se han situado fuera del Derecho (positivismo extremo), y a veces se han visto los valores como el centro del Derecho (Derecho natural). En el futuro, también en relación con este dilema, tendremos ante nosotros la tercera vía, en la que los valores tienen más significado de lo que un positivista desearía darles, pero en la que 
los valores tratan también de evitar los peligros del Derecho natural. Aquí, las obras de Carlos Santiago Nino tienen mucho que enseñamos.

Todo esto lo he dicho con grandes reservas. Me gustaría citar de nuevo a Georg Henrik von Wright: El búho de Minerva ha alzado el vuelo. Comienza su vuelo al atardecer. En el desarrollo social, esto significa que lo anterior camina irreversiblemente hacia su fin, pero somos incapaces de ver con claridad qué es lo nuevo que reemplazará a lo viejo. Sólo podemos estar seguros de que en el futuro la filosofía jurídica será tan necesaria como hoy o incluso de manera más aguda. Las respuestas existen «en algún lado»; sólo necesitamos encontrar las preguntas correctas. Las nuevas generaciones deben buscar persistentemente esas cuestiones.

M. A.: Durante esta entrevista, has afirmado varias veces que no consideras ya a la teoría de la argumentación jurídica como un campo de especial interés. Sin embargo, si, como dices, se produce "la dirección de la sociedad por medios democráticos», ¿no significa eso que necesitaremos un uso creciente de medios argumentativos? Y en cualquier caso, ¿a qué se debe esa pérdida de interés en la teoría de la argumentación jurídica?

A. A.: Al mencionar que mi interés en la teoría de la argumentación jurídica ha disminuido, no quiero decir que niegue el valor de esa teoría. Sólo he remarcado que mis posibilidades para el desarrollo técnico de esa teoría están simplemente agotadas. O quizás deba decir que he formulado las bases de mi teoría de la argumentación de una manera que resulta suficiente considerando cuáles son los propósitos que hoy en día considero necesita la teoría. Como cuestión de hecho, mi atención se dirige cada vez más hacia la función social y la influencia de la teoría de la argumentación.

En mi propio país, he trabajado durante varios años en el desarrollo del razonamiento de las decisiones judiciales. Puesto que Finlandia es un pequeño país, cabe decir que la teoría jurídica ha tenido éxito en sus esfuerzos, al menos de forma razonable. Los tribunales de justicia han internalizado mucho de lo que se ha presentado, por ejemplo, en los libros «Interpreting Statutes» (1991) e «Interpreting Precedentes» (1997), ambos editados por Neil MacCormick y Robert S. Summers. En mi opinión, la naturaleza de este cambio social es un interesante objeto de estudio. Aquí, es una cuestión de cambios en la cultura jurídica y de evolución cultural en un sentido importante, pero aún no específico, de la expresión.

El desarrollo que ha tenido lugar en los tribunales de justicia se relaciona con el cambio en la estructura de las normas. Éste parece ser un fenómeno bien conocido casi en todas las democracias occidentales, y también en Finlandia. Con ello me refiero al incremento de la textura abierta y de las formas flexibles y, en general, de las normas vinculadas con principios (por ejemplo, principios que se refieren a derechos humanos). Ahora que esta regulación 
se ha vuelto más abierta de esta forma, ha surgido por el lado de la interpretación tradicional del Derecho o incluso más del pasado la necesidad de sopesar y buscar equilibrios. Y donde esto ocurre, el fenómeno es socialmente importante. Éste es el fenómeno real que ha empezado a interesarme más que el desarrollo técnico de la teoría de la argumentación en la que, por ejemplo, Robert Alexy y Aleksander Peczenik han efectuado un excelente trabajo.

En relación con el significado general de la teoría de la argumentación, entiendo su función en el mismo sentido en que entiendo el significado de la filosofía en general. Es cuestión de formular los conceptos por medio de los cuales uno puede entender su propia acción mejor que antes. La teoría de la argumentación es, por ejemplo, un instrumento de los jueces para una menor autocomprensión. Y cuando es éste el caso, la teoría también puede, al menos indirectamente, influir en cambiar la práctica social. Es cuestión de incrementar el conocimiento práctico aristotélico o, más correctamente, de incrementar el «know-how»y el cambio en la acción a través suyo.

M. A.: Dada cual ha sido tu evolución en la teoría del Derecho, ¿te consideras a ti mismo como un filósofo del Derecho analítico? ¿Cuáles son, en tu opinión, los rasgos típicos de un iusfilósofo analítico?

A. A.: A final de la década de los 60 y comienzos de los 70, mi concepción de la teoría del Derecho se basaba en la filosofía de Georg Henrik von Wright y Alf Ross. Era analítica en un sentido relativamente estricto de la expresión.

Sin embargo, más adelante -debido a los seminarios de von Wright- me interesé en la filosofía del último Wittgenstein. A este respecto, podría caracterizar mi concepción jurídica desde el final de los 60 también como analítica, pero sólo en el sentido en que las «Investigaciones filosóficas» de Wittgenstein representan la filosofía analítica. Este giro hace también comprensible por qué se volvió muy importante para mí la nueva retórica de Perelman. Él estaba buscando precisamente la «tercera vía» entre la (o además de) la inducción y la deducción.

Como consecuencia de ello, durante los últimos 30 años no he sido un representante del positivismo, que frecuentemente va asociado con la filosofía analítica. Por el contrario, me he separado claramente de él; donde más claramente aparece es en «Lo racional como razonable», pero incluso antes, esta separación resulta evidente en muchos de mis artículos. En este punto, mi pensamiento difiere claramente, por ejemplo, del tipo de teoría que representa Eugenio Bulygin, aunque tengo una gran estimación por mi buen amigo Eugenio en su calidad de teórico. Hay un aspecto que permite reflejar adecuadamente esta distinción. En mi concepción, la racionalidad discursiva ocupa una posición prominente. Sin embargo, el positivismo estrictamente 
analítico no reconoce a la racionalidad discursiva como forma de pensamiento.

En algún momento, Aleksander Peczenik ha llamado a este tipo de aproximación, que -creo- también yo represento, neorrealismo, de cara a distinguirlo del realismo (escandinavo) tradicional. Pongo un ejemplo para ilustrar la distinción. Cuando la filosofía analítica ha estudiado la ciencia jurídica (por ejemplo, Alf Ross y, también, Hans Kelsen) suele empezar definiendo lo que es ciencia. La respuesta de tipo kelseniano es no-empirista y la rossiana empirista. Personalmente, deseo separarme de ese tipo de aproximación. Pone a la ciencia jurídica en el lecho de Procusto (le corta la cabeza y las piernas para que la ciencia jurídica pueda encajar en el lecho de la ciencia). En mis estudios, el punto de partida lo ha constituido la ciencia jurídica como tal, que yo mismo he cultivado como profesor a tiempo completo de Derecho civil. Esta ciencia jurídica, que es ostensiblemente predominante en los países nórdicos, no es ni puramente analítica ni puramente empírica. Desarrolla su tarea social, por un lado, sistematizando las normas jurídicas y, por otro lado, haciendo recomendaciones normativas.

A este respecto, no es muy interesante saber si la ciencia jurídica cumple las características de alguna ciencia «establecida» o no. Lo esencial es estudiar cómo se sistematizan las normas y de qué manera se controlan las interpretaciones de la ciencia jurídica. En esta forma, se vuelven importantes los conceptos de sistema normativo y de sistematización. Cuando se considera el control de las interpretaciones, la teoría de la argumentación es esencial, porque esa teoría no se cuestiona si los enunciados son verdaderos o no, sino que se cuestiona si los puntos de vista pueden razonarse o no en forma creíble. Esta aproximación ofrece esencialmente instrumentos más ricos que los que suministra el positivismo analítico estricto para evaluar las condiciones teóricas que conciernen al control de los enunciados de la ciencia jurídica.

Creo que el cambio en el pensamiento de mi maestro y amigo Georg Henrik con Wright durante las dos últimas décadas ha sido indicativo también de la perspectiva que he asumido en mi trabajo. Estoy enormemente interesado en sus puntos de vista acerca de la naturaleza de la filosofía, el mito del progreso y de las consecuencias que, por ejemplo, tiene la teoría de la acción desde el punto de vista social. Algunos de mis escritos representan un esfuerzo por reflexionar sobre estas interpretaciones en el campo del Derecho.

M. A.: Estamos ahora terminando el siglo XX. En tu opinión, ¿cuáles son los nombres principales, los clásicos, de la filosofía del Derecho de este siglo? 
A. A.: Uno espera que este tipo de elenco de grandes pensadores ha de ser largo, pues cualquier propuesta de lista excluirá a alguna persona significativa e influyente. Sin embargo, ahora trato de animarme y daré sólo una lista muy limitada.

Sin ninguna duda, considero a Georg Henrik von Wright como el mayor filósofo del Derecho. Su contribución a la lógica deóntica y, por medio de ella, a una rama de la filosofía jurídica analítica es quizás su mayor logro. Además, sus escritos ilustrando la última fase de Wittgenstein y, por ejemplo, su trabajo «Explicación y comprensión» han tenido una gran influencia también en la filosofía no analítica.

Además de a von Wright, naturalmente considero a Hans Kelsen, Alf Ross y H. L. A. Hart como grandes filósofos del Derecho, y lo mismo se aplica a Chaïm Perelman, Norberto Bobbio y Axel Hägerström. Por lo que se refiere a mi trabajo teórico, también han sido muy importantes Carlos Alchourrón, Otto Brusiin, Eugenio Bulygin, Ernesto Garzón Valdés, Kazimierz Opalek y Jerzy Wróblewski. Desde la década de los 80, muchos pensadores que representan al mundo de habla hispana me han impresionado grandemente. Como uno de ellos, menciono aquí a Francisco Laporta.

Considerando los cambios que han tenido lugar en mi trabajo teórico en las dos últimas décadas, naturalmente, no puedo dejar de incluir en la lista a Jürgen Habermas y Jaakko Hintikka. En cuanto a representantes de mi misma generación, deseo mencionar a Robert Alexy y Aleksander Peczenik, que han influido en mí con sus teorías, pero que son también colegas próximos y amigos queridos, como lo es Garzón Valdés y lo fue también Wróblewski.

M. A.: A propósito del tema de los valores, has sugerido una tercera vía, entre el positivismo extremo y el Derecho natural. ¿Podrías ser más preciso sobre tu concepción del Derecho e ilustrarla quizás con algún ejemplo que muestre cómo funciona en la práctica?

A. A.: El positivismo extremo no toma en cuenta el hecho de que cuando la estructura de las normas está dada en forma abierta y los principios se vuelven cada vez más importantes en el pensamiento jurídico, también la relación entre el Derecho y la moral (o los valores en general) cambiará. Fácilmente podemos señalar casos en los que la interpretación del Derecho está inevitablemente teñida de valores, y sin embargo decimos que se trata de una cuestión de interpretación del Derecho y no meramente de una cuestión de juicio moral. Es el caso, por ejemplo, cuando tenemos que sopesar y balancear los principios del Tratado Europeo de Derechos Humanos o los derechos básicos incluidos en una Constitución.

Sin embargo, no he aprobado nunca la concepción del Derecho natural según la cual el Derecho «natural», es decir, la moral, es el criterio último 
del Derecho; en otras palabras, que la naturaleza jurídica del Derecho válido (ius positivum) estaría determinada por la moral. Aleksander Peczenik ha defendido una versión dulcificada al sugerir que la demanda de observancia de las reglas jurídicas es una exigencia moral. En mi opinión, no tenemos necesariamente que pensar de esa forma. He tratado de dar mi propia respuesta a ese dilema en mi libro «Lo racional como razonable», donde estudio varios tipos de conceptos relativos a la validez y, entre otras cosas, la función de la llamada «Grundnorm».

Dicho brevemente, mi punto de partida es que todo Derecho es primariamente Derecho dado (Derecho positivo). Los códigos (o las valoraciones) morales en cuanto tales, no es asunto jurídico. Cuando uno dice «No debes matar», ha formulado un juicio moral. En algunas situaciones, una actitud (valoración) moral tiene lugar «dentro» del Derecho. Es el caso, por ejemplo, cuando uno interpreta las leyes que se refieren al aborto por razones sociales (el aborto, sobre esa indicación socioeconómica, es legal en Finlandia). Uno interpreta el Derecho, pero inevitablemente utilizará un argumento moral o tendrá que ordenar puntos de vista contradictorios en un orden de preferencia basado en valoraciones. El Derecho y la moral se entrelazan. Esto ocurre constantemente en la aplicación cotidiana del Derecho y, sin embargo, no podemos decir que cuando los jueces aplican el Derecho sólo argumentan moralmente. 
DOXA 21-I (1998) 